\title{
Comparison of silicone versus polyurethane ureteral stents: a prospective controlled study
}

\author{
Nariman Gadzhiev1, Dmitry Gorelov', Vigen Malkhasyan², Gagik Akopyan³, Revaz Harchelava ${ }^{3}$ Denis Mazurenko ${ }^{4}$, \\ Christina Kosmala ${ }^{5}$, Zhamshid Okhunov ${ }^{5^{*}}$ (i) and Sergei Petrov ${ }^{1}$
}

\begin{abstract}
Background: Approximately $80 \%$ of patients with indwelling ureteral stents experience stent related symptoms (SRS). We believe SRS can be reduced through altering the composition of ureteral stents to a less firm material. Therefore, we aim to compare modern silicone and polyurethane ureteral stents in terms of SRS intensity and safety.

Methods: From June 2018 to October 2018, patients from two distinct clinical centers were prospectively enrolled in the study and stratified (non-randomly) into either control group A, patients who received polyurethane stents (Rüsch, Teleflex), or experimental group B, patients who received silicone stents (Cook Medical). Each participant completed a survey $1 \mathrm{~h}$ after stent insertion, in the middle of the stent dwelling period, and before stent removal or ureteroscopy noting body pain and overactive bladder via the visual analog scale pain (VASP) and overactive bladder $(\mathrm{OAB})$ awareness tool, respectively. Additionally, successfulness of stent placement, hematuria, number of unplanned visits, and stent encrustation rates were assessed within each group.
\end{abstract}

Results: A total of 50 patients participated in the study, control group A consisted of 20 patients and experimental group B consisted of 30 patients. Participants in group B, silicone ureteral stents, demonstrated significantly lower mean values of VASP 2 weeks prior to stent removal and promptly before stent removal $(p=0.023$ and $p=0.014$, respectively). No other comparisons between the two groups were statistically significant.

Conclusions: Compared to polyurethane ureteral stents, silicone ureteral stents are associated with lower body pain intensity assessed by VASP 2 weeks before stent removal and the time of stent removal.

Trial registration: Current Controlled Trials NCT04000178. Retrospectively registered on June 26, 2019.

Keywords: Ureteral stent, Silicone, Polyurethane, Stent-related symptoms, Quality of life

\section{Background}

Double-J ureteral stents have become a fundamental endourological treatment since Roy P. Finney introduced them in 1978 [1]. In the United States, approximately 92,000 ureteral stents are placed annually to manage upper urinary tract obstructions caused by urolithiasis and other genitourinary pathologies [2]. While most indwelling stents aid patients with drainage, over $80 \%$ of patients with indwelling stents experience stent related

\footnotetext{
* Correspondence:

${ }^{5}$ Department of Urology, University of California, Irvine, 333 City Boulevard West, Orange, CA 92868, USA

Full list of author information is available at the end of the article
}

symptoms (SRS), such as storage symptoms and pain, which lower their quality of life (QoL) [3, 4]. Several studies have been conducted comparing different variations of stents characteristics and the impact each had on patients experiencing SRS. Through these studies, physicians can better individualize ureteral stents for each patient in order to decrease SRS. These characteristics include the length of the stent [5], stent positioning [6], changing bladder pig tail to loop type [7], use of stents with special coatings [8], and composition of biomaterials of different firmness [9]. Additionally, the use of alpha1- and choline blockers, with their combinations [10], nonsteroidal antiinflammatory drugs [11] and

(c) The Author(s). 2020 Open Access This article is distributed under the terms of the Creative Commons Attribution 4.0 International License (http://creativecommons.org/licenses/by/4.0/), which permits unrestricted use, distribution, and reproduction in any medium, provided you give appropriate credit to the original author(s) and the source, provide a link to the Creative Commons license, and indicate if changes were made. The Creative Commons Public Domain Dedication waiver (http://creativecommons.org/publicdomain/zero/1.0/) applies to the data made available in this article, unless otherwise stated. 
mirabegron [12] have also been studied in order to reduce intensity of SRS experienced by patients. Despite this, patients with indwelling stents continue to experience SRS [13].

In a study by Lennon et al., a significant increase in SRS was attributed to ureteral stent firmness [12]. The magnitude of stent firmness is reliant on the material ureteral stents are composed of. Today, ureteral stents are primarily composed of polyurethane due to a low fracture propensity and high tensile strength [14]. However, when ureteral stents were first introduced in 1978, they were composed of silicone [15]. Compared to modern polyurethane ureteral stents, silicone ureteral stents were softer, more biocompatible [16], and have lower encrustation rates [17]. Despite these benefits, silicone ureteral stents were ultimately replaced with polyurethane ureteral stents due to higher frictional forces during placement [15] and smaller side holes as a result of lower tensile strength. However, technological advancements have made it possible to produce silicone ureteral stents similar to polyurethane ureteral stents. With these advancements we believe that adopting modern silicone ureteral stents could reduce SRS and as a result increase patient's quality of life (QOL) with indwelling ureteral stents. This study aims to compare patient discomfort between modern silicone and polyurethane ureteral stents.

\section{Material and methods}

After institutional review board approval (IRB), informed consent was obtained from 50 patients admitted with acute renal colic from June 2018 till October 2018 at two academic institutions. Inclusion criteria included age (18 to 60 years old), confirmed ureteral stone and prescribed ureteral stent placement for pain syndrome relief. Patients were excluded from the study if they had an active urinary tract infection. Participating patients were then divided into two groups: group A $(n=20)-$ patients who received polyurethane stents (Rüsch, Teleflex) - and group B $(n=30)$ - patients who received silicone stents (Cook Medical). All patients received $6 \mathrm{Fr}$, $26 \mathrm{~cm}$ ureteral stents and were placed via cystoscopy and X-ray guided control under total intravenous anesthesia with propofol and fentanil. Stent indwelling time lasted for 4 weeks during which each patient underwent a follow-up assessment $1 \mathrm{~h}$ after insertion, in the middle of the stent dwelling period, and before ureteroscopy or stent removal.

\section{Outcome measurement}

The primary outcome of this study was the assessment of body pain and bladder irritation. Secondary outcomes of the study were success of stent placement, hematuria, unplanned visits and stent encrustation, defined by urologist at the time of the stent removal. At each follow-up appointment, patients completed a survey in which the visual analog scale pain (VASP) and overactive bladder $(\mathrm{OAB})$ awareness tools were used to assess the intensity of SRS. Both of these tools have been previously validated for body pain and overactive bladder symptoms assessment $[18,19]$.

The ureteral stent symptom questionnaire (USSQ), which is considered as a gold standard for evaluating SRS, was not used since there is no validated Russian version.

\section{Statistical analysis}

For all parameters, 95\% confidence intervals (CIs) were calculated. To calculate CIs for the differences and/or ratio, the MOVER approach was used implemented in the spreadsheets: MOVER-D.xls and MOVER-R.xls (http://profrobertnewcomberesources.yolasite.com/). To present interval estimations the compact format was used in which the lower and upper limits are shown as subscripts surrounding the point estimate [20]. PAST software was used to test the agreement of the observed values with the normal distribution and for the statistical estimation of the parameters and their comparisons [21]. For the analysis of discrete data the exact nonparametric methods were used implemented in StatXact package (http://www.cytel.com/software/statxact). Boxplots with whiskers and notches were drawn using online BoxPlotR (http://shiny.chemgrid.org/boxplotr/). A $p$-value of < 0.05 was considered statistically significant.

\section{Results}

A total of 50 patients underwent stent insertion and were stratified as follows: 20 pts. received polyurethane stents and 30 pts. silicone stents. Their demographic and clinical data are presented in Table 1. Both groups appeared to be statistically homogeneous except the stone size, which was statistically larger in Polyurethane group $(p=0.001)$, and we didn't regard it as relevant to the aim of the study.

Table 1 Demographic and clinical data

\begin{tabular}{llll}
\hline & Polyurethane & Silicone & $p$-value \\
\hline Patients, n (\%) & $20(40 \%)$ & $30(60 \%)$ & \\
Age (years); median (range) & $50(19-60)$ & $48(24-64)$ & 0.96 \\
Male, n (\%) & $7(35 \%)$ & $18(60 \%)$ & 0.19 \\
Female, n (\%) & $13(65 \%)$ & $12(40 \%)$ & \\
BMl (kg/m $\left.{ }^{2}\right)$ & $2626_{31}$ & 262830 & 0.71 \\
Stone size (S) (mm) & $912_{13}$ & ${ }_{6} 8_{10}$ & $\mathbf{0 . 0 0 1}$ \\
Stent installation time, min & 6.17 .89 .4 & 6.87 .88 .9 & 1.00 \\
Duration of stay of the stent, weeks & 3.23 .53 .7 & 3.63 .84 .0 & 0.015 \\
\hline
\end{tabular}

$p$-values in the last column are for the comparisons between groups 
Comparison of VASP and OAB awareness tool at $1 \mathrm{~h}$ after stent insertion, in the middle of the stent dwelling period and before ureteroscopy or stent removal (Table 2) demonstrated significant differences between mean values of VASP at 2 weeks and before stent removal in favor of group B (silicone ureteral stents) ( $p=$ 0.023 and $p=0.014$, respectively).

In order to specify the difference between the groups score totals were calculated and compared (Table 3). Mean VASP scores in Silicone group were significantly lower than in Polyurethane group $(p=0.0010)$. It should be noted that polyurethane stents lead to an abnormally high variance in scores in comparison to silicone stents. This means that the results with the polyurethane stents appeared to be more variable, uncertain and unpredictable than with the silicone stents.

No statistically significant difference was observed between groups comparing secondary outcomes (Table 4).

\section{Discussion}

In 1967, Dr. Paul Zimskind first reported using ureteral silicone tubing as an indwelling stent. In theory, these stents were to provide aid with drainage for up to 19 months however, some were expelled due to the absence of a mechanism to prevent stent migration [22]. Seven years later, Gibbons presented a silicone stent equipped with a distal flange and sharply pointed barbs to prevent upward and downward migration, respectively. Nonetheless, this design was difficult to insert into patients because the barbs increased the stent size from 7 Ch to 11 Ch [2]. Then in 1978, Roy P. Finney presented the double pigtail silicone stent design that most resembles the ureteral stents used in practice today.

Ureteral stents are mainly used to relieve urinary obstruction and are used in a variety of cases such as

Table 2 Comparison of VAS Pain, OAB awareness tool and EQ5D-5 $\mathrm{L}$ questionnaire at three points: $1 \mathrm{~h}$ and 2 weeks after insertion, and before stent removal

\begin{tabular}{|c|c|c|c|}
\hline \multicolumn{4}{|c|}{ VASP, scores } \\
\hline $1 \mathrm{~h}$ & 1.42 .84 .0 & 1.42 .02 .5 & 0.23 \\
\hline 2 weeks & 1.32 .43 .4 & $0.71 .1_{1.5}$ & 0.023 \\
\hline Before & 1.32 .12 .9 & 0.81 .11 .4 & 0.014 \\
\hline$p$-value & 0.25 & 0.0029 & \\
\hline \multicolumn{4}{|c|}{ OAB, scores } \\
\hline $1 \mathrm{~h}$ & 4.27 .210 & 4.76 .37 .8 & 0.61 \\
\hline 2 weeks & 5.18 .211 & 4.86 .27 .2 & 0.25 \\
\hline Before & 5.78 .712 & 5.06 .88 .5 & 0.27 \\
\hline$p$-value & 0.48 & 0.76 & \\
\hline
\end{tabular}

Data are shown as medians; the lower and upper limits of $95 \% \mathrm{Cl}$ are given as left and right subscripts. $p$-values in the last column are for the comparisons between groups; $p$-values in rows are from ANOVA for the repeated measurements at three-time points. Statistically significant values are in bold obstructing stones, strictures, and aid in effective drainage [23]. Despite their benefits, over $80 \%$ of patients with indwelling ureteral stents experience stent-related pain that affects their ability to perform daily activities, causing at least one-third of patients to prematurely remove their ureteral stents [24]. Premature removal of ureteral stents can alternatively affect patient recovery and lead to more post-operative complications. Thus, to improve surgical outcomes and patient health, it is important to limit SRS. In a study conducted by Lennon et al. [25], it was concluded that the softness of the ureteral stent directly influenced patients' tolerability; stents made from softer material correlated with less incidence of dysuria and pain [15]. Additionally, in a recent study found that silicone ureteral stents were associated with less patient discomfort at day 20 Post OP [26]. Therefore, in order to further reduce SRS, alterations to stent composition could be a solution.

In this study, the intensity of SRS in traditional polyurethane ureteral stents and modern silicone ureteral stents were compared. From the data, use of the $\mathrm{OAB}$ awareness tool proved unfit to accurately assess patient QoL. However, VASP data concluded that patients who received silicone ureteral stents had a significantly better QoL in the middle of the stent indwelling period and immediately prior to stent removal compared to those who received polyurethane ureteral stents $(p=0.023$ and $p=0.014$, respectively).

Despite these findings, some studies have not found a correlation between stent material composition and patient QoL [27-29]. Due to these inconsistent findings, additional long-term research studies should be completed before any conclusive statements can be made regarding stent material and the affect it has on QoL. Nonetheless, none of these studies specifically compared silicone ureteral stents to polyurethane stents. This could be mainly due to recent advancements in modern technology that have made it possible to manufacture silicone ureteral stents with the same external diameter, internal diameter, and size of side holes as in polyurethane ureteral stents. Due to these advancements our study displayed the superiority of silicone ureteral stents in terms of body pain at 2 weeks before stent removal and immediately before stent removal.

Comparing stent-related complications from both Group A and Group B, no significant difference was observed. These results speak to the overall safety of silicone ureteral stents. Originally, silicone ureteral stents were replaced with polyurethane ureteral stents due to low tensile strength which limited the internal diameter and aperture of side holes. Additionally, silicone ureteral stents proved more difficult to place due to a high volume of friction and were more expensive to produce leading to the switch to polyurethane ureteral stents 
Table 3 Comparison of the score totals of VASP and OAB in the patients with the polyurethane and silicone stents

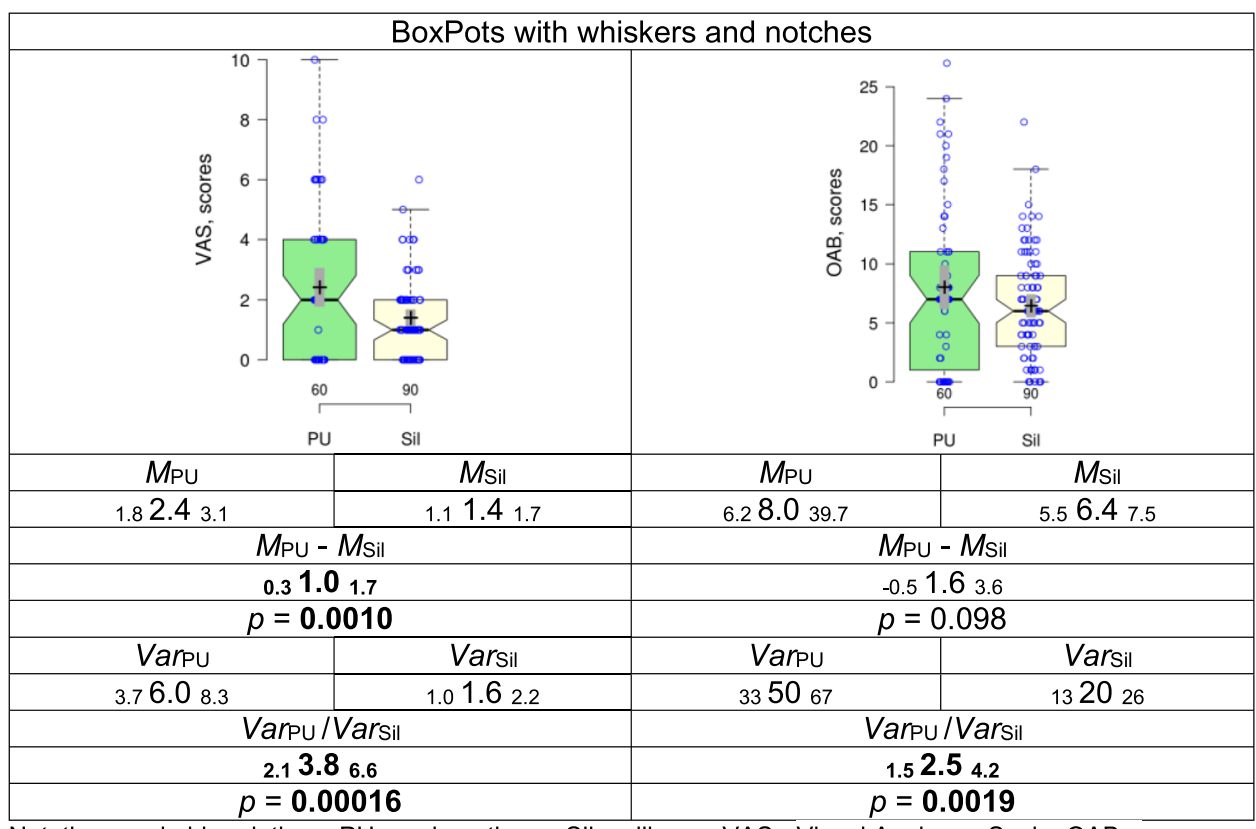

Notations and abbreviations: PU - polyurethane, Sil - silicone, VAS - Visual Analogue Scale, OAB -

OverActive Bladder, $M$ - mean, Var - variance. The lower and upper limits of $95 \% \mathrm{Cl}$ are given as left and right subscripts.

Notations and abbreviations: PU polyurethane, Sil silicone, VAS Visual Analogue Scale, OAB OverActive Bladder, $M$ mean, Var variance. The lower and upper limits of $95 \% \mathrm{Cl}$ are given as left and right subscripts

[30]. Because of recent technological advancements, silicone stents are beginning to become more similar to traditional polyurethane ureteral stents in both size and safety. In our study there was no significant difference in the stent encrustation rate. This could have been due to the small sample size used and short duration of the study limited to 4 weeks. A larger sample size and longer study duration should be used to accurately determine if stent encrustation would differ between polyurethane and silicone ureteral stents.

Table 4 Secondary outcomes comparison between groups

\begin{tabular}{|c|c|c|c|}
\hline \multirow{2}{*}{$\begin{array}{l}\text { Secondary } \\
\text { outcome }\end{array}$} & \multicolumn{2}{|l|}{ Stent } & \multirow{2}{*}{$\begin{array}{l}p- \\
\text { value }\end{array}$} \\
\hline & Polyurethane & Silicone & \\
\hline \multicolumn{4}{|c|}{ Difficult stent placement } \\
\hline No & $13(64 \%)$ & $26(85 \%)$ & 0.09 \\
\hline Yes & $7(36 \%)$ & $4(15 \%)$ & \\
\hline \multicolumn{4}{|c|}{ Unplanned visits } \\
\hline No & $17(83 \%)$ & $30(100 \%)$ & 0.052 \\
\hline Yes & $3(17 \%)$ & $0(0 \%)$ & \\
\hline \multicolumn{4}{|c|}{ Encrustation } \\
\hline No & 17 (83\%) & $29(95 \%)$ & 0.29 \\
\hline Yes & $3(17 \%)$ & $1(5 \%)$ & \\
\hline \multicolumn{4}{|l|}{ Hematuria } \\
\hline No & $9(45 \%)$ & $19(63 \%)$ & 0.25 \\
\hline Yes & $11(55 \%)$ & $11(37 \%)$ & \\
\hline
\end{tabular}

Thus, silicone ureteral stents may be a viable option for patients who have had a previous negative experience with polyurethane ureteral stents or could alternatively be used as a replacement stent for patients with current indwelling polyurethane ureteral stents experiencing SRS. However, silicone ureteral stents depending on country can be more expensive than polyurethane ureteral stents as in Russia, which can be an obstacle receiving such stents.

Our study did have some limitations: it was a nonrandomized study with a small sample size which may have affected our study results. Additionally, groups differed in terms of stone size which could possibly affect successfulness of stent placement. One of the instruments for assessment of SRS was OAB awareness tool, which is not very popular but has Russian validated translation. Further research is needed to confirm the results of our study.

\section{Conclusions}

Silicone ureteral stents were associated with lower body pain intensity assessed by VASP 2 weeks prior to stent removal and immediately prior to stent removal in comparison with polyurethane ureteral stents.

\section{Abbreviations}

OAB: Overactive bladder; PU: Polyurethane; QoL: Quality of life; SRS: Stent related symptoms; VASP: Visual analog scale pain 


\section{Acknowledgements}

To Nikita Khromov-Borisov for helping in statistical analysis.

\section{Authors' contributions}

NG, DG, DM, ZO and SP - Study conception \& design and manuscript writing; VM and GA - Data collection and analysis; RH and CK - Manuscript writing and editing. All authors have read and approved the final manuscript. All authors have made significant contribution to the manuscript.

\section{Funding}

None. There was no funding to support this study or publication.

\section{Availability of data and materials}

The datasets used and/or analyzed during the current study are available from the corresponding author on reasonable request.

\section{Ethics approval and consent to participate}

Local ethics committee (Pavlov First Saint Petersburg State Medical University, Russia) approval was obtained to conduct this study. All patients were consented to participate in the study. Specifically written informed consent was obtained from the participants. There are no patient identifying information in the manuscript.

\section{Consent for publication}

Not applicable.

\section{Competing interests}

The author(s) declare(s) that they have no competing interests. Dr. Zhamshid Okhunov, as a member of the editorial board (Section Editor) of this journal, had no role in the handling this manuscript.

\section{Author details}

'Department of Urology, Pavlov First Saint Petersburg State Medical University, Saint Petersburg, Russia. ${ }^{2}$ Department of Urology, State University of Medicine and Dentistry, Moscow, Russia. ${ }^{3}$ Institute for Urology and Reproductive Health, Sechenov University, Moscow, Russia. ${ }^{4}$ Department of Urology, llyinskaya Hospital, Moscow, Russia. ${ }^{5}$ Department of Urology, University of California, Irvine, 333 City Boulevard West, Orange, CA 92868, USA

Received: 10 June 2019 Accepted: 20 January 2020

Published online: 03 February 2020

\section{References}

1. Finney RP. Experience with new double J ureteral catheter stent. J Urol. 1978;120:678-81.

2. Donahue RP, Stamm AW, Gibbons RP, et al. Evolution of the ureteral stent: the pivotal role of the Gibbons ureteral catheter. Urology. 2018;115:3-7.

3. Joshi HB, Okeke A, Newns $\mathrm{N}$, et al. Characterization of urinary symptoms in patients with ureteral stents. Urology. 2002;59:511-6.

4. Liu Q, Liao B, Zhang R, et al. Combination therapy only shows short-term superiority over monotherapy on ureteral stent-related symptoms outcome from a randomized controlled trial. BMC Urol. 2016;16:66.

5. Pilcher JM, Patel U. Choosing the correct length of ureteric stent: a formula based on the Patient's height compared with direct ureteric measurement. Clin Radiol. 2002;57:59-62.

6. Miyaoka R, Monga M. Ureteral stent discomfort: etiology and management. Indian journal of urology: IJU: journal of the Urological Society of India. 2009;25:455-60.

7. Kawahara $\mathrm{T}$, Ito $\mathrm{H}$, Terao $\mathrm{H}$, et al. Changing to a loop-type ureteral stent decreases patients' stent-related symptoms. Urol Res. 2012:40:763-7.

8. Riedl CR, Witkowski M, Plas E, Pflueger H. Heparin coating reduces encrustation of ureteral stents: a preliminary report. Int J Antimicrob Agents. 2002:19:507-10.

9. Mosayyebi A, Vijayakumar A, Yue QY, et al. Engineering solutions to ureteral stents: material, coating and design. Central Eur J Urol. 2017:70:270-4.

10. Gao Y, Liang H, Liu L et al. Comparison of alpha-blockers and Antimuscarinics in improving ureteral stent-related symptoms: a metaanalysis. Urol J. 2019;16(3):307-311.
11. Tadros NN, Bland $L$, Legg $E$, et al. A single dose of a non-steroidal antiinflammatory drug (NSAID) prevents severe pain after ureteric stent removal: a prospective, randomised, double-blind, placebo-controlled trial. BJU Int. 2013;111:101-5.

12. Tae BS, Cho S, Jeon BJ, et al. Does mirabegron relieve ureteric stent-related discomfort? A prospective, randomized, multicentre study. BJU Int. 2018;122: 866-72.

13. Yoshida $T$, Inoue $T$, Taguchi M, et al. Efficacy and safety of complete intraureteral stent placement versus conventional stent placement in relieving ureteral stent-related symptoms: A randomized, prospective, single-blinded, multicenter clinical trial. J Urol. 2019:202(1):164-170.

14. Gorman SP, Jones DS, Bonner MC, et al. Mechanical performance of polyurethane ureteral stents in vitro and ex vivo. Biomaterials. 1997;18:137983

15. Venkatesan N, Shroff S, Jayachandran K, Doble M. Polymers as ureteral stents. J Endourol. 2010;24:191-8.

16. Denstedt JD, Wollin TA, Reid G. Biomaterials used in urology: current issues of biocompatibility, infection, and encrustation*. J Endourol. 1998;12:493500.

17. Beiko DT, Knudsen BE, Denstedt JD. Advances in ureteral stent design. J Endourol. 2003;17:195-9.

18. K. S. Coyne, T. Zyczynski, M. K. Margolis, V. Elinoff, and R. G. Roberts, "Validation of an overactive bladder awareness tool for use in primary care settings.," Adv Ther, 22, no. 4, pp. 381-394.

19. Bijur PE, Silver W, John Gallagher E. EDUCATIONAL ADVANCES reliability of the visual analog scale for measurement of acute pain; 2001

20. Louis TA, Zeger SL. Effective communication of standard errors and confidence intervals. Biostatistics. 2009:10:1-2

21. Hammer $\varnothing$, Harper DAT, Ryan PD. Paleontological statistics software package for education and data analysis. Palaeontol Electron. 2001;4:9-18.

22. Zimskind PD, Fetter TR, Wilkerson JL. Clinical use of long-term indwelling silicone rubber ureteral splints inserted cystoscopically. J Urol. 1967;97:840-

23. Rao MV, Polcari AJ, Turk TM. Updates on the use of ureteral stents: focus on the resonance ${ }^{\circledast}$ stent. Med Devices (Auckl). 2010:4:11-5.

24. Joshi HB, Stainthorpe A, Macdonagh RP, et al. Indwelling ureteral stents: evaluation of symptoms, quality of life and utility. J Urol. 2003:169:1065-9.

25. Lennon GM, Thornhill JA, Sweeney PA, et al. "Firm" versus "soft" double pigtail ureteric stents: a randomised blind comparative trial. Eur Urol. 1995; 28:1-5.

26. Wiseman O, Letendre J, Cloutier J, et al (2018) Effects of silicone hydrocoated double loop ureteral stent on symptoms and quality of life in patients undergoing FURS for kidney stones a comparative randomized multicenter clinical study.

27. Hb J, Sv C, Nagarajan M, et al. A prospective randomized single-blind comparison of ureteral stents composed of firm and soft polymer. J Urol. 2005;174:2303-6.

28. Bregg K, Riehle RA. Morbidity associated with indwelling internal ureteral stents after shock wave lithotripsy. J Urol. 1989;141:510-2.

29. Pryor $\mathrm{L} L$, Langley MJ, Jenkins $A D$. Comparison of symptom characteristics of indwelling ureteral catheters. J Urol. 1991;145:719-22.

30. Tunney MM, Keane PF, Gorman SP. Assessment of urinary tract biomateria encrustation using a modified Robbins device continuous flow model. Biomed Mater Res. 1997;38:87-93.

\section{Publisher's Note}

Springer Nature remains neutral with regard to jurisdictional claims in published maps and institutional affiliations. 